${ }^{3}$ Thang Hoa General Hospital, Quang Nam Province, Vietnam

${ }^{4}$ Social Insurance of Danang City, Vietnam

\title{
Gestational diabetes diagnosed in third trimester of pregnancy: an observation at a Hospital of Women and Children in Vietnam
}

\section{ABSTRACT}

Background. Gestational diabetes mellitus (GDM) remains a significant concern within the medical community due to its high risk, as well as its serious side effects on both the mothers and the fetuses. This study aims to assess the prevalence and the risk factors of gestational diabetes mellitus in pregnant women at Da Nang Hospital for Women and Children.

Methods. A cross-sectional study was conducted on 706 pregnant women at 2428 weeks of gestation at Da Nang hospital to determine the prevalence of gestational diabetes. Multivariate regression analysis was used to clarify the independent risk factors associated with gestational diabetes. All participants were interviewed and-tested for the oral glucose tolerance test (OGTT) to identify the number of gestational diabetes, which was diagnosed according to the American Diabetes Association (ADA) diagnostic criteria in 2014. Results. Gestational diabetes prevalence was $10.2 \%$; categorized by the number of matched diagnostic criteria: 1 criterion: $7.1 \%$; 2 criteria: $2.1 \%$; 3 criteria: $1.0 \%$. There are four independent risk factors for gestational diabetes determined through multivariate regression analysis: maternal age $>30$ years $(O R=2.376)$,

Address for correspondence:

Tran Thua Nguyen

Department of General Internal

Medicine and Geriatrics

Hue Central Hospital

16 Le Loi street, Hue city, Vietnam

e-mail: tranthuanguyen23@gmail.com

Clinical Diabetology 2020, 9; 6: 411-415

DOI: $10.5603 /$ DK.2020.0055

Received: 15.07.2020

Accepted: 02.11.2020 a history of gestational diabetes (OR $=12.211)$, prepregnancy $\mathrm{BMI} \geq 23 \mathrm{~kg} / \mathrm{m}^{2}(\mathrm{OR}=10.775)$, a history of fetal macrosomia $>3800 \mathrm{~g}(\mathrm{OR}=4.655)$. The risk of gestational diabetes in the group with risk factors was 6.21 times higher than that in the group with no risk factors. Conclusion. More attention should be paid to the risk factors for gestational diabetes, such as maternal age $>\mathbf{3 0}$ years, a history of gestational diabetes, pre-pregnancy $B M I \geq 23 \mathrm{~kg} / \mathrm{m}^{2}$, a history of fetal macrosomia $>3800 \mathrm{~g}$ in all pregnant women. (Clin Diabetol 2020; 9; 6: 411-415)

Key words: gestational diabetes mellitus, risk factors, prevalence

\section{Introduction}

Gestational diabetes or gestational diabetes mellitus (GDM) is a condition in which diabetes is diagnosed during pregnancy that is not clearly overt diabetes. Gestational diabetes mainly occurs during the $24^{\text {th }}-28^{\text {th }}$ week of pregnancy, when a fetus produces a great number of hormones that prevent insulin receptors from functioning properly and disturbs the blood sugar levels.

Generally, gestational diabetes manifests few symptoms, and it is most commonly diagnosed by screening during pregnancy. Diagnostic tests detect inappropriately high levels of glucose in blood samples. Gestational diabetes accounts for 9.6 to 13 percent of all pregnancies [1].

The risk of perinatal mortality does not increase while the risk of fetal macrosomia does. Other perinatal risks include shoulder dystocia, birth injuries such as 
bone fractures and nerve palsies, and hypoglycemia. Long-term adverse health outcomes which are reported among infants born by mothers with gestational diabetes include sustained impairment of glucose tolerance, subsequent obesity (although not when adjusted for size), and impaired intellectual achievement. For women, gestational diabetes is a strong risk factor for diabetes. GDM carries risks for both the mother and her neonates. The risks associated with gestational diabetes are well recognized, and there is a certain treatment to lower maternal glucose levels in order to reduce these risks [2].

Along with the growth of diabetes; comes the continuing spread rate of gestational diabetes mellitus (GDM). At the moment, GDM remains a significant concern within the medical community due to its high risk, as well as its side effects on both the mothers and the fetuses. It is also the reason why the American Diabetes Association (ADA) in 2014 has issued the diagnostic criteria to manage and prevent GDM's complications [3]. We designed this study to assess the prevalence and risk factors of gestational diabetes mellitus in pregnant women at Da Nang Hospital for Women and Children.

\section{Methods}

\section{Study population}

From January 2018 to March 2019, consecutive women who had either a singleton or twin pregnancy with gestation between 24 and 28 weeks (third trimester) at Da Nang Hospital for Women and Children were enrolled in this study. They visited the Obstetric Department for a regular examination. Women with previously treated gestational diabetes or active chronic systemic disease (except essential hypertension) were excluded.

The present study was approved by the ethics committee of Hue University of Medicine and Pharmacy, Vietnam. All participants were provided written informed consent. Subjects were provided with written information about the study and were briefed orally again before their oral glucose tolerance test. People whose glucose levels exceeded cut-off values for eligibility were diagnosed with gestational diabetes.

\section{Oral glucose tolerance test}

A 75-g oral glucose tolerance test (OGTT) was performed in women at 24-28 weeks of gestation who were not previously diagnosed with overt diabetes. Plasma glucose was measured when the patient is fasting and at 1 and 2 hours after the test. The OGTT was performed in the morning after an overnight fast of at least eight hours. The diagnosis of GDM is made when one or more following plasma glucose value are met or exceeded, according ADA 2014 guidelines: 1) Fasting: $\geq 92 \mathrm{mg} / \mathrm{dL}$ (5.1 mmol/L); 2) first hour: $\geq 180$ $\mathrm{mg} / \mathrm{dL}(10.0 \mathrm{mmol} / \mathrm{L}) ; 3)$ second hour: $\geq 153 \mathrm{mg} / \mathrm{dL}$ (8.5 mmol/L) [3].

All participants were advised to follow a 48 hours normal diet before the oral glucose tolerance test and to fast for 8 hours the night before the test. Blood samples were obtained after the overnight fast and one and two hours after the receipt of the 75-g oral glucose load.

\section{Outcome variables}

Clinical outcomes among the women included: maternal age, gestational age at birth, birth weight, and body mass index (BMI).

We explored some of the risk factors such as GDM medical history; maternal age; a family medical history of type 1 diabetes; pre-pregnancy BMI; a history of fetal macrosomia and pathological obstetrics (e.g. stillbirth, miscarriage); polycystic ovary syndrome.

\section{Statistical analysis}

Statistical analyses were based on the SPSS software, version 16.0. Continuous variables were analyzed by means if they were normally distributed and by medians of nonparametric tests if their distribution was abnormal. A P value of 0.05 was considered to indicate statistical significance.

\section{Results}

From January 2018 to March 2019, oral glucose tolerance test (OGTT) was performed in 706 pregnant women at Da Nang Hospital for Children and Women. According to ADA 2014 diagnostic criteria, in this study, GDM accounted for 72 (10.2\%). Among pregnancies affected by GDM, according to ADA 2017, the group with $\geq 1$ criterion accounted for $7.1 \%$ (the highest percentage).

In bivariate analysis, factors correlated with GDM were maternal age $\geq 30$ years (OR 1.8,95\%Cl 1.1-2.9, $\mathrm{P}$ $=0.02) ; \mathrm{BMI} \geq 23 \mathrm{~kg} / \mathrm{m}^{2}$ (OR 10.8, 95\% Cl 6.3-18.4, $\mathrm{P}<$ $0.001)$ and a history of fetal macrosomia $>3800 \mathrm{~g}$ (OR 5.2, 95\% Cl 2.7-10.2, P < 0.001) (Table 1). With multivariable regression, there were independent risk factors (IRFs) of GDM indicated in this research, including GDM history, pre-pregnancy $\mathrm{BMI} \geq 23 \mathrm{~kg} / \mathrm{m}^{2}$; a history of fetal macrosomia $>3800 \mathrm{~g}$ (as shown in Table 2). The percentage of previous birth weight $>3800 \mathrm{~g}$ and maternal age $>30$ years were highest $(37.8 \%$ and $37.1 \%$ ) (Fig. 1). The group with risk factors was more likely to suffer from GDM than the group without those, statistical significance differentiation (14.6 vs. 2.7, P < 0.001) (Table 3). 
Table 1. Bivariate analysis of the relationship between risk factors and GDM

\begin{tabular}{|c|c|c|c|c|}
\hline Variable & Gestational diabetes mellitus $\mathrm{n}(\%)$ & No gestational diabetes mellitus $\mathrm{n}(\%)$ & OR $(95 \% \mathrm{Cl})$ & $P$ value \\
\hline \multicolumn{5}{|c|}{ Age $\geq 30$ years } \\
\hline Yes & $36(13.7 \%)$ & $226(86.3 \%)$ & $1.8(1.1-2.9)$ & 0.02 \\
\hline No & $36(8.1 \%)$ & $408(91.9 \%)$ & & \\
\hline \multicolumn{5}{|c|}{$\mathrm{BMI} \geq 23 \mathrm{~kg} / \mathrm{m}^{2}$} \\
\hline Yes & $47(33.3 \%)$ & $94(66.7 \%)$ & $10.8(6.3-18.4)$ & $<0.001$ \\
\hline No & $25(4.4 \%)$ & $540(95.6 \%)$ & & \\
\hline \multicolumn{5}{|c|}{ History of macrosomia $>3800 \mathrm{~g}$} \\
\hline Yes & $35(18.8 \%)$ & $151(81.2 \%)$ & $5.2(2.7-10.2)$ & $<0.001$ \\
\hline No & $13(4.2 \%)$ & $293(95.8 \%)$ & & \\
\hline
\end{tabular}

Table 2. Multivariable regression of risk factors associated with gestational diabetes mellitus

\begin{tabular}{lcccc}
\hline Risk factors & Regression coefficient & $\mathbf{P}$ & OR & The interval where OR $=95 \%$ \\
\hline GDM history & 2.502 & $<0.001$ & 12.211 & $3.29-45.28$ \\
Maternal age $>30$ & 0.865 & 0.016 & 2.376 & $1.17-4.81$ \\
Pre-pregnancy BMI & & & & \\
$\geq 23 \mathrm{~kg} / \mathrm{m}^{2}$ & 2.377 & $<0.001$ & 10.775 & $5.27-22.00$ \\
History of macrosomia $>3800 \mathrm{~g}$ & 1.538 & $<0.001$ & 4.655 & $2.24-9.68$ \\
\hline
\end{tabular}

*Does not include cases with first-time pregnancies; ${ }^{* *}$ does not include cases with twin or more pregnancies

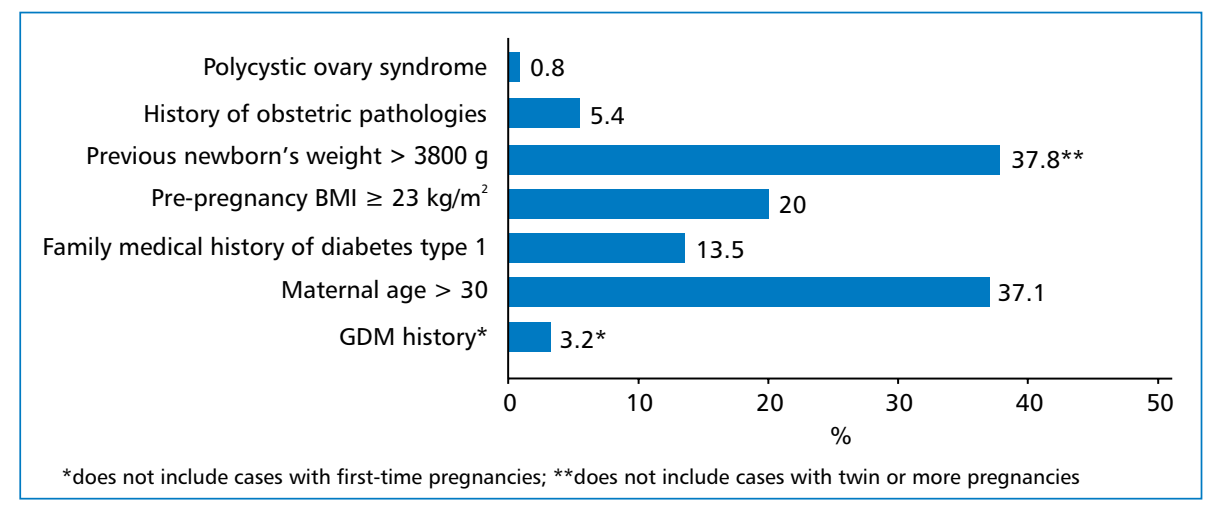

Figure 1. The percentage with respect to independent risk factors

Table 3. The percentage of affected GDM between the group with and without independent risk factors

\begin{tabular}{|c|c|c|c|c|c|}
\hline \multirow[t]{2}{*}{$\begin{array}{l}\text { Risk } \\
\text { factors }\end{array}$} & \multirow[t]{2}{*}{ Number } & \multicolumn{2}{|c|}{$\begin{array}{c}\text { Gestational } \\
\text { diabetes mellitus }\end{array}$} & \multicolumn{2}{|c|}{$\begin{array}{l}\text { No gestational } \\
\text { diabetes mellitus }\end{array}$} \\
\hline & & $n$ & $\%$ & $n$ & $\%$ \\
\hline Yes & 445 & 65 & 14.6 & 380 & 85.4 \\
\hline No & 261 & 7 & 2.7 & 254 & 97.3 \\
\hline $\mathrm{P} ; \mathrm{OR}$ & & \multicolumn{4}{|c|}{$\mathrm{P}<0.001 ; \mathrm{OR}=6.21$} \\
\hline
\end{tabular}

\section{Discussion}

Prevalence of GDM

In this study, the incidence of GDM was $10.2 \%$, a figure similar to the $10.6 \%$ found in the Vietnamese population in Australia [4]. Our findings on prevalence of GDM are fairly similar to a recent study that reported the prevalence of GDM in 8 Eastern and Southeast Asian countries - 10.1\% (95\% Cl 6.5-15.7) [1]. However, the prevalence of GDM in our study was higher than that in 
some studies in Vietnam. Thao et al carried out a study in 415 pregnancies (pregnant women) at Bach Mai Hospital showed that the proportion of GDM was 7.9\% based on ADA 2003 diagnostic criteria [5]. A research undertaken by $\mathrm{Nga}$ in 2009 in 1327 pregnant women at Bach Mai Hospital and Central Maternity Hospital demonstrated the percentage of GDM was 7.8\% [6].

The prevalence of GDM in our study was found to be lower when compared with Chinese women who live in China (13.7\%) [7] or in Australia (13.9\%) [8]. A metaanalysis included 84 studies from 20 Asian countries. They demonstrated the prevalence of GDM was $11.5 \%$ (95\% Cl 10.9-12.1) [9]. We have no clear reason for this difference, but we speculate that it may due to maternal age and BMI disparities, as well as ethnic background [10].

The epidemiological studies aim to determine the prevalence of GDM in a community, which is important to design effective screening strategies, improve the risk factors and manage effectively on pregnant women with hyperglycemia. These benefits reflected the increased use of induction of labor for the mothers and the increased rate of admission to the neonatal nursery for the infants, both of which may be depend on the experience of the physicians. The earlier gestational age at birth, as a result of the induction of labor, may have contributed to the reduction in serious perinatal outcomes. Others have reported an increased rate of cesarean delivery associated with the diagnosis and treatment of gestational diabetes.

\section{Risk factors of GDM}

The risk factors of GDM were analyzed in this current review. Multiparity $\geq 2$, a previous history of GDM, congenital anomalies, stillbirth, abortion, preterm delivery, macrosomia, having concurrent pregnancy-induced hypertension, polycystic ovary syndrome, age $\geq 25$, BMI $\geq 25$, and a family history of diabetes are the significant risk factors predictive of GDM in current pregnancy (OR values ranged from 1.90 to 8.42 ). Most of the guidelines, including those of ADA in 2016, recommend universal screening for GDM in second trimester [11]. According to the American Maternity Association, maternal age $\geq 25$ years were considered the average risk factors for GDM. Meanwhile, the Australasian Diabetes in Pregnancy Society (ADIPS) originally recommended that pregnant women aged over 40 years were at highrisk of GDM [12].

Our study showed that those with a history of previous GDM, pre-pregnancy BMI $\geq 23 \mathrm{~kg} / \mathrm{m}^{2}$ and a history of fetal macrosomia $>3800 \mathrm{~g}$ are more likely to develop GDM compared those without a history of these conditions, respectively. This finding is consistent with previous studies. Idris et al. conducted the study in 366 Malaysian women showing that the rate of GDM in the age groups $<24,25-35, \geq 35$ years was: $3 \%$; $14,6 \% ; 38.6 \%$ respectively [13]. Studying risk factors in pregnant women in Asia, Wagaarachchi found that the prevalence of GDM among women aged $\geq 35$ years was $7.8 \%, 2.5$ times higher than in the age group < 35 years, at $3.1 \%$ [2]. According to the study carried out by Rajput in 2011 in 607 pregnant women diagnosed with GDM, the percentage of GDM in patients aged 25 years and older is 3.8 times than that in the group below 25 years old [14]. Yang's research (2009) in 16286 pregnant women has proven the statistically significant difference between the groups of age $\geq 35$ and < 35 (OR: 1.97, P < 0.001) [15]. In our research, the percentage of GDM in the group over 30 years old was higher than that in the group 30 and below $(13.7 \%$ vs. $8.1 \%, P<0.05)$. The odds ratio of GDM between the group $>30$ years old and group $\leq 30$ was 1.8 . All the above results come to a general conclusion that the rate of GDM tends to increase with age.

Weight loss at birth was both a consequence of GDM and a risk factor for postpartum pregnancy. Europeans consider a baby with a birth weight $\geq 4000$ $\mathrm{g}$ to be large for gestational age (LGA); in Vietnam, a baby with a birth weight $\geq 3600 \mathrm{~g}$ can be considered to be LGA. Therefore, a history of fetal macrosomia was one of the risk factors for GDM, because increased blood glucose levels go through the placenta causing increased glucose concentration in the fetus and large fetal weight. Insulin had an anabolic effect that stimulates growth either directly or indirectly through growth factors.

All of these warned that the incidence of high-risk pregnancies in the future would increase, and doctors should be aware of the high-risk factors for pregnant women during antenatal care and screening for early detection of GDM.

\section{Conclusion}

The prevalence of GDM is rather high (10.2\%). The risk factors associated with GDM were identified: maternal age ( $\geq 30$ years) and a history of macrosomia. Overall, the ratio of GDM between groups with and without independent risk factors was 6.21.

\section{Conflict of interest}

The authors declare no conflict of interest. 


\section{REFERENCES}

1. Nguyen $C L$, Pham NM, Binns CW, et al. Prevalence of gestational diabetes mellitus in Eastern and Southeastern Asia: a systematic review and meta-analysis. J Diabetes Res. 2018; 2018: 6536974, doi: 10.1155/2018/6536974, indexed in Pubmed: 29675432.

2. Wagaarachchi PT, Fernando L, Premachadra P, et al. Screening based on risk factors for gestational diabetes in an Asian population. J Obstet Gynaecol. 2001; 21(1): 32-34, doi: 10.1080/01443610020022087, indexed in Pubmed: 12521908.

3. American Diabetes A. Standards of medical care in diabetes 2014. Diabetes Care. 2014; 37(Supplement ): S14.

4. Cheung NW, Wasmer G, Al-Ali J. Risk factors for gestational diabetes among Asian women. Diabetes Care. 2001; 24(5): 955-956, doi: 10.2337/diacare.24.5.955, indexed in Pubmed: 11347764 .

5. Thao N, Nga VB. Determining the prevalence of gestational diabetes and investigating the risk factors of pregnant women managing pregnancy in Bach Mai Hospital's obstetrics department. Vietnam Medical Journal. 2007; 27(3): 135-139.

6. Nga VB. Researching fasting blood glucose threshold for screening gestational diabetes and initial assessing of treatment effectiveness. Vietnam Medical Journal. 2009; 29(2): 319-322.

7. Lao TT, Tam KF. Gestational diabetes diagnosed in third trimester pregnancy and pregnancy outcome. Acta Obstet Gynecol Scand. 2001; 80(11): 1003-1008, doi: 10.1034/j.16000412.2001.801106.x, indexed in Pubmed: 11703196.

8. Beischer NA, Oats JN, Henry OA, et al. Incidence and severity of gestational diabetes mellitus according to country of birth in women living in Australia. Diabetes. 1991; 40 Suppl 2: 35-38, doi: 10.2337/diab.40.2.s35, indexed in Pubmed: 1748263.

9. Lee KW, Ching SM, Ramachandran V, et al. Prevalence and risk factors of gestational diabetes mellitus in Asia: a systematic review and meta-analysis. BMC Pregnancy Childbirth. 2018; 18(1): 494, doi: 10.1186/s12884-018-2131-4, indexed in Pubmed: 30547769.

10. Yuen L, Wong VW. Gestational diabetes mellitus: Challenges for different ethnic groups. World J Diabetes. 2015; 6(8): 1024-1032, doi: 10.4239/wjd.v6.i8.1024, indexed in Pubmed: 26240699.

11. American Diabetes A. 2. Classification and Diagnosis of Diabetes: Standards of Medical Care in Diabetes - 2020. Diabetes Care. 2020; 43(Suppl 1): S14-S31, doi: 10.2337/dc20-S002, indexed in Pubmed: 31862745.

12. Australian Diabetes in Pregnancy Society. ADIPS consensus guidelines for the testing and diagnosis of gestational diabetes mellitus in Australia. 2013.

13. Idris N, Hatikah C, Murizah MZ. Universal versus selective screening for detection of gestational diabetes mellitus in a Malaysian population. Malays Fam Physician. 2009; 4(2-3): 83.

14. Rajput R, Yadav $Y$, Nanda S, et al. Prevalence of gestational diabetes mellitus \& associated risk factors at a tertiary care hospital in Haryana. The Indian journal of medical research. 2013; 137(4): 728.

15. Yang $H$, Wei $Y$, Gao $X$, et al. China National GDM Survey Working Group. Risk factors for gestational diabetes mellitus in Chinese women: a prospective study of 16,286 pregnant women in China. Diabet Med. 2009; 26(11): 1099-1104, doi: 10.1111/j.14645491.2009.02845.x, indexed in Pubmed: 19929987. 